\title{
Sound-insulation layers low-frequency modeling, using the fuzzy structure theory
}

\author{
Laurent Gagliardini, Charles Fernandez \\ PSA-Peugeot-Citroën, France \\ Christian Soize \\ Université Paris-Est, France
}

\begin{abstract}
Over the past few years, car manufacturers have improved their numerical models for the prediction of the trimmed body vibroacoustic response. In the lowfrequency band $[20,200] \mathrm{Hz}$, sound-insulation layer modeling remains a critical topic. Recent work allows the connection of the structure and cavity through a transfer matrix computed from a FE model of the soundinsulation layer. Nevertheless, such an approach requires a $\mathrm{FE}$ model of sound-insulation layer, which may not be available in early design stages. Moreover, considering the uncertainty of the design itself, in addition to material uncertainty, a deterministic model may not be appropriate. In this paper, a simplified model of sound-insulation layers based on the fuzzy structure theory is proposed. The simplified model is obtained by performing a statistical averaging of the internal dynamical degrees of freedom of the sound-insulation layer and is governed by three parameters: the modal density, the coefficient of participating mass and the damping rate of the sound-insulation layer. In order to improve the prediction, the model errors introduced by the simplifications as well as material properties uncertainty are modeled using a nonparametric probabilistic approach. After the fuzzy model is set up theoretically, it is applied to a simplified case. The way how the fuzzy model's parameters are related to design parameters will then be discussed. Finally, the application to a full trimmed body is presented.
\end{abstract}

\section{INTRODUCTION}

Trimmed body modeling have been extensively used in the past 10 years to design car bodies versus lowfrequency $([20-200 \mathrm{~Hz}])$ noise issues such as booming or rolling noise. Nevertheless insulating devices modeling remains an open topic since their standard modeling is a lumped mass distribution whereas they are known to have resonances around $100 \mathrm{~Hz}$. One of the reasons for this poor modeling is just that detailed finite element modeling of trimming material would lead to a large increase of the number of vibration eigenmodes, that is not compatible with the industrial numerical process. It would also require great model construction efforts.

In the same time, insulating technologies have evolved to answer OEM requirements on mass reduction. Efficient solutions have been designed regarding the main goal of insulating devices, that is to say, high frequencies. But it appears that such lightweight insulation also changes the trimmed body low- and medium-frequency vibroacoustic responses. Recent software developments now allow finite element modeling including up-to-date material modeling. Once more, the model construction efforts as well as computational resources are important. Specifically, such models do not apply at the early stage of a project when most of the critical decisions are made.

The aim of this paper is to introduce an efficient modeling of mass-spring insulating systems [1]. By efficient, we mean here requiring minimum modeling efforts and computational resources to address a given problem. Let us then consider the modeling problem of mass-spring insulating devices.

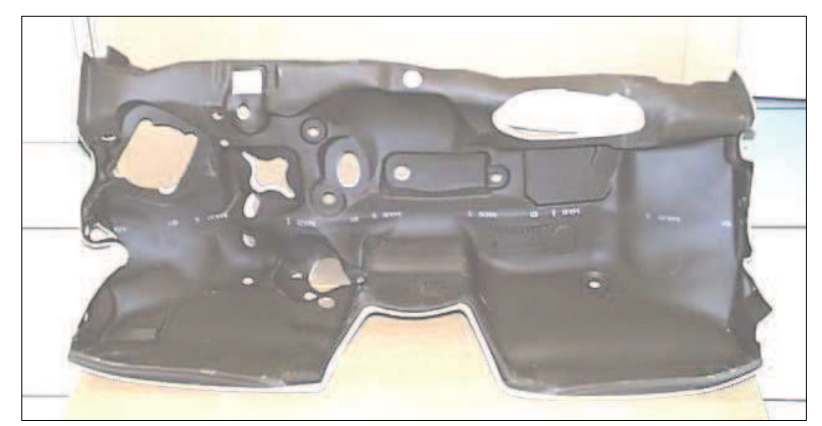

Figure 1: Picture of a sound-insulation device (dash trim) 


\section{TRIM MODELING PROBLEM ANALYSIS}

Figure 1 shows a dash insulator. It is made of a thin and heavy layer set over thicker and softer foam - or fibrous - layer. It not a well-defined object since its thickness is changing all over the dash to meet packaging constraints and porous material is known to be strongly heterogeneous. Moreover, the exact connection between the foam and the supporting structure is also uncertain. Finally, the trim instant design is subject to changes during the vehicle design process. In such an uncertain context, a probabilistic method seems appropriate.

From the vibroacoustic point of view, the dash trim is a high modal density component. The modal analysis of a single dash trim model, using typical properties, leads to more than 20,000 modes up to $600 \mathrm{~Hz}$. In such a context, the dynamic hypersensitivity avoids any modal interpretation. Nevertheless, from our own experience, statistics of the modal behavior -e.g. the modal densityappear to be rather stable, allowing useful practical developments.

At this point, the idea of modeling the dash trim as a random distribution of single degrees of freedom comes rather naturally. This idea fits quite well with the fuzzy structure theory $[2,3]$ introduced by one of the authors in the late 80's as a probabilistic model of numerous uncertain degrees of freedom connected to a so-called master structure and already used in structural-acoustics problems [4]. In this paper, the individual degrees of freedom constituting the fuzzy structure will be considered as a physical model of the actual massspring system. This will allow estimating the skin displacement and later the vibroacoustic coupling with the internal cavity.

Even if the fuzzy structures theory was developed to compute mean values as well as fluctuations statistics, the strategy used below is to keep only the mean values of the dynamical responses. Fluctuations can be computed using a nonparametric probabilistic method that will not be detailed in this paper. See Refs. $[5,6,7,8]$ for further information.

The background and direction of the work beeing settled, let's come back to the mechanical-mathematical problem to be solved.

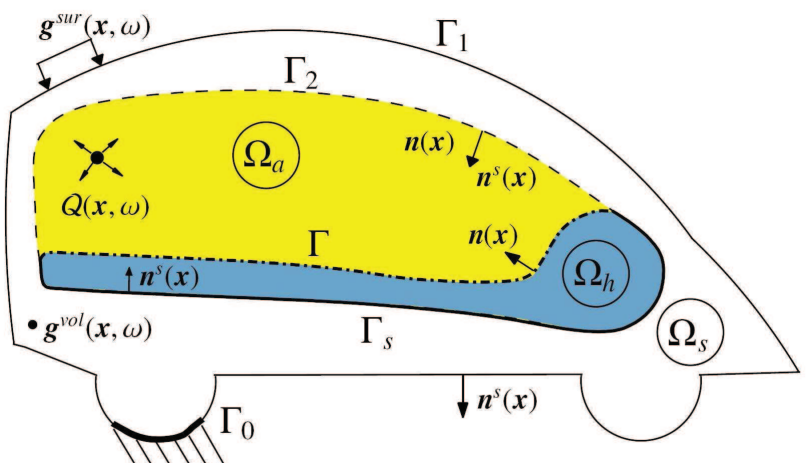

Figure 2: structural-acoustics problem definition

\section{VIBROACOUSTIC PROBLEM INVOLVING TRIM MATERIAL GENERAL FORMULATION}

Figure 2 defines the geometry of the problem to be treated involving 3 bounded domains: the structural part, $\boldsymbol{\Omega}_{S}$, the internal cavity, $\boldsymbol{\Omega}_{a}$, and the trim material, $\boldsymbol{\Omega}_{h}$. Boundaries of these domains are partitioned in order to separate external boundary conditions from loading surfaces and interaction surfaces. Three main interaction surfaces are considered: $\Gamma_{2}$ between the structural part and the cavity (standard vibroacoustic interaction), $\Gamma_{\mathrm{s}}$ between the structural part and the trim (mechanical interaction) and $\Gamma$ between the trim and the inner cavity (vibroacoustic interaction).

The problem variables are the displacements field of the structural part, $u_{s}$, the acoustic pressure field inside the cavity, $p$, and the displacement field of the trim material, $u_{h}$. The general boundary value problem is discretized using finite elements, leading to a linear system $[3,9,8]$, such as:

$$
\left[\begin{array}{cccc}
{\left[\underline{A}_{i i}^{S}\right]} & {\left[\underline{A}_{i j}^{S}\right]} & {\left[\underline{A}_{i f}^{S}\right]} & {[0]} \\
{\left[\underline{A}_{j i}^{S}\right]} & {\left[\underline{A}_{j j}^{S}\right]} & {\left[\underline{A}_{j f}^{S}\right]} & {\left[\underline{C}_{j}\right]} \\
{\left[\underline{A}_{f i}^{S}\right]} & {\left[\underline{A}_{f j}^{S}\right]} & {\left[\underline{A}_{f f}^{S}\right]} & {\left[\underline{C}_{f}\right]} \\
{[0]} & \omega^{2}\left[\underline{C}_{j}\right]^{T} & \omega^{2}\left[\underline{C}_{f}\right]^{T} & {\left[\underline{A}^{F}\right]}
\end{array}\right]\left[\begin{array}{c}
\underline{\boldsymbol{u}}_{i}^{S} \\
\underline{\boldsymbol{u}}_{j}^{S} \\
\underline{\boldsymbol{u}}_{f}^{S} \\
\underline{\boldsymbol{p}}^{F}
\end{array}\right]=\left[\begin{array}{c}
\underline{\boldsymbol{f}}^{S}(\omega) \\
\underline{\boldsymbol{f}}_{S}^{H}(\omega) \\
0 \\
\underline{\boldsymbol{f}}^{F}(\omega)+\underline{\boldsymbol{f}}_{F}^{H}(\omega)
\end{array}\right]
$$

The structural displacements are classically projected over the eigenmodes $\left[\underline{\Phi}^{s}\right]$ of the structural part in vacuo, and acoustic pressures are projected over the eigenmodes $\left[\underline{\Phi}^{a}\right]$ of the cavity with rigid walls. Generalized coordinates are introduced such as:

$$
\underline{u}^{s}(\omega)=\left[\underline{\Phi}^{s}\right] \underline{q}^{s}(\omega) \quad, \quad \underline{p}(\omega)=\left[\underline{\Phi}^{a}\right] \underline{q}^{a}(\omega)
$$

After elimination of the trim degrees of freedom, the following modified vibroacoustic formulation is obtained:

$$
\begin{aligned}
& \left.\left(\begin{array}{cc}
{\left[\underline{\mathbb{A}}^{S}(\omega)\right]} & {\left[\underline{\mathbb{C}}_{f}\right]} \\
\omega^{2}\left[\underline{\mathbb{C}}_{f}\right]^{T} & {\left[\underline{\mathbb{A}}^{F}(\omega)\right]}
\end{array}\right]+\left[\begin{array}{cc}
{\left[\underline{\mathbb{B}}^{S}(\omega)\right]} & {\left[\underline{\mathbb{B}}^{C}(\omega)\right]} \\
\omega^{2}\left[\underline{\mathbb{B}}^{C}(\omega)\right] & {\left[\underline{\mathbb{B}}^{F}(\omega)\right]}
\end{array}\right]\right)\left[\begin{array}{l}
\underline{\boldsymbol{q}}^{S} \\
\underline{\boldsymbol{q}}^{F}
\end{array}\right] \\
& =\left[\begin{array}{l}
\underline{\mathbb{E}}(\omega)^{S} \\
\underline{\mathbb{E}}(\omega)^{F}
\end{array}\right]
\end{aligned}
$$

with

$$
\begin{aligned}
& {\left[\underline{\mathbb{B}}^{S}(\omega)\right]=\left[\underline{\Downarrow}_{j}^{S}\right]^{T}\left(\left[\underline{A}_{j j}^{H}\right]-\left[\underline{A}_{j i}^{H}\right]\left[\underline{A}_{i i}^{H}\right]^{-1}\left[\underline{A}_{i j}^{H}\right]\right)\left[\underline{\Psi}_{j}^{S}\right]} \\
& {\left[\underline{\mathbb{B}}^{F}(\omega)\right]=-\omega^{2}\left[\underline{\Phi}^{F}\right]^{T}\left(\left[\underline{C}_{j}\right]^{T}\left[\underline{A}_{i i}^{H}\right]^{-1}\left[\underline{C}_{j}\right]\right)\left[\underline{\Phi}^{F}\right]} \\
& {\left[\underline{\mathbb{B}}^{C}(\omega)\right]=\left[\underline{\Downarrow}_{j}^{S}\right]^{T}\left(-\left[\underline{A}_{j i}^{H}\right]\left[\underline{A}_{i i}^{H}\right]^{-1}\left[\underline{C}_{j}\right]\right)\left[\underline{\Phi}^{F}\right]}
\end{aligned}
$$


This formulation accounts for the trim as a modification of both structural and acoustic dynamic stiffness, as well as coupling matrices. The size of the numerical problem is unchanged compared to a standard vibroacoustic problem, but such a formulation requires building the frequency dependent matrices accounting for the trim effects on the structural part, acoustic cavity and coupling terms. This formulation is very close to the one proposed in Refs. $[9,10,11]$.

\section{FUZZY-STRUCTURE MODELING OF THE TRIM WITHIN THE VIBROACOUSTIC MODEL}

In this section, the fuzzy structure theory is used to build an alternate simplified formulation of the vibroacoustic problem. Figure 3 defines the proposed simplified modeling, involving random oscillators. For this purpose, it will be assumed that the interaction between the trim and the structural part is limited to the normal components of forces and displacements. Moreover, as far as trimming systems thickness is small compared to acoustical and structural wavelengths, domains $\Gamma_{\mathrm{s}}$ and $\Gamma$ as well as their respective normal, $\mathbf{n}^{\mathrm{s}}$ and $\mathbf{n}$, are assumed to be identical. After proper derivations and averaging (See Ref [1]), the above generalized balance equation of the vibroacoustic system is transformed into a mean reduced coupled problem:

$$
\begin{aligned}
& {\left[\begin{array}{cc}
{\left[\underline{A}^{s}(\omega)\right]+\underline{a}^{s}(\omega)\left[\underline{B}^{s}\right]} & {[\underline{C}]+\underline{a}^{c}(\omega)\left[\underline{C}^{s}\right]} \\
\omega^{2}\left\{[\underline{C}]^{T}+\underline{a}^{c}(\omega)\left[\underline{C}^{s}\right]^{T}\right\} & {\left[\underline{A}^{a}(\omega)\right]+\underline{a}^{a}(\omega)\left[\underline{B}^{a}\right]}
\end{array}\right]\left[\begin{array}{l}
\underline{\boldsymbol{q}}^{s}(\omega) \\
\underline{\boldsymbol{q}}^{a}(\omega)
\end{array}\right]} \\
& =\left[\begin{array}{l}
\underline{\boldsymbol{f}}^{s}(\omega) \\
\underline{\boldsymbol{f}}^{a}(\omega)
\end{array}\right]
\end{aligned}
$$

where $a^{s}, a^{c}, a^{a}$ are scalar expressions computed with the fuzzy-structure parameters,

The matrices $\left[B^{s}\right],\left[B^{a}\right]$ and $\left[C^{s}\right]$ are constant matrices of spatial coupling between the model components; they are computed from specific bilinear forms.

Fluctuations around the mean model are then introduced using a random matrix theory - also called nonparametric modeling ref. [5] - for $\left[B^{s}\right],\left[B^{a}\right]$ and $\left[C^{s}\right]$ (see the details in [1]).

The fuzzy-structure parameters are mean values of some physical properties of the underlying model:

- Mean modal density, $n(\omega)$,

- $\quad$ Mean participating mass, $m(\omega)=m_{0} v(\omega)$ with $m_{0}$ the physical mass of the component and $v(\omega)$ a coefficient of participating mass $(0<v(\omega) \leq 1)$,

- Mean damping coefficient $\xi(\omega)$.
These 3 parameters are a key-point when applying the fuzzy structure theory, since they capture the mean dynamical behavior of the whole insulating device. The next section is dedicated to their determination.

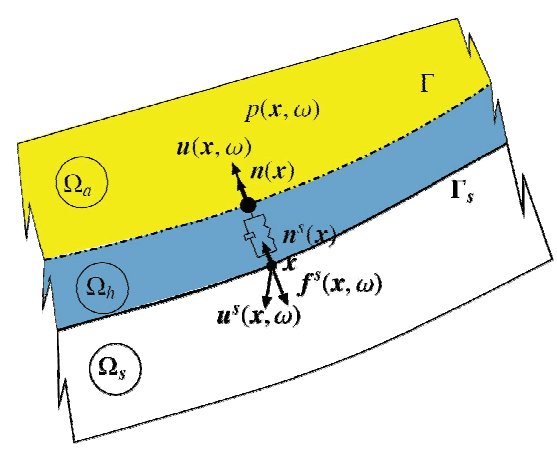

Figure 3: Simplified trim modeling using a fuzzy single degree of freedom system.

\section{FUZZY PARAMETER COMPUTATION}

As any simplified theory, the fuzzy structure theory involves dedicated parameters that have to be fitted.

Depending on the modeling issues (vibratory or vibroacoustic analysis), various methodologies can be proposed for an optimal application of the theory. In this paper, we present a method relying on a FE mesh of the insulating layer. This mesh can be the actual mesh of the component, when available, or just a simplified mesh with relevant material properties and geometry. The mesh is constrained (clamped) on the foam surface, since this boundary condition is the closest to the actual

configuration.

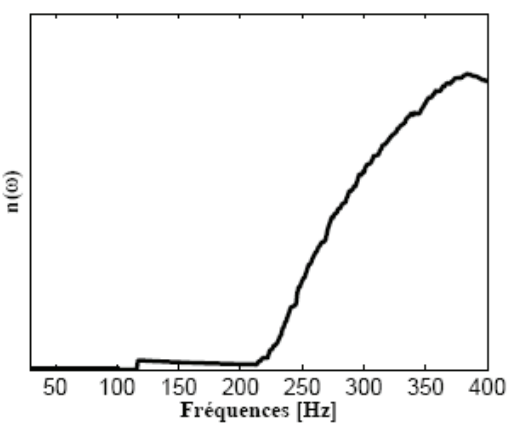

Figure 4: Mean modal density of a dash trim, computed from an isolated FE model

\section{MODAL DENSITY}

The modal density is computed from the numerical modal extraction. Contributing modes - i.e. modes with their main displacements along the normal - are separated from "in-plane" modes that cannot be described by the proposed model. The modal density, $n(\omega)$, is then computed using a smoothing procedure.

Figure 4 shows the typical modal density computed for a dash trim. It may be noticed that the first normal elastic 
modes arise above $110 \mathrm{~Hz}$, and that the modal density begins to grow significantly above $200 \mathrm{~Hz}$.

\section{PARTICIPATING MASS}

The coefficient of participating mass is computed such as the kinetic energy of the heavy layer may, in average, be approximated by:

$$
\left\langle E_{c}(\omega)\right\rangle_{B}=m(\omega)\left\langle v^{2}\right\rangle_{\Gamma, B}
$$

After appropriate algebraic calculations and smoothing, the participating mass is computed as a function of the the modal shapes of the selected participating modes (See [12]).

Figure 5 shows the graph of the coefficient of participating mass for the dash insulator. It can be noticed that when the modal density is null, the coefficient of participating mass is one, meaning that the trim behaves as an added lumped mass. For higher frequencies, the coefficient of participating mass decreases. At the moment, there is no clear physical interpretation of this result.

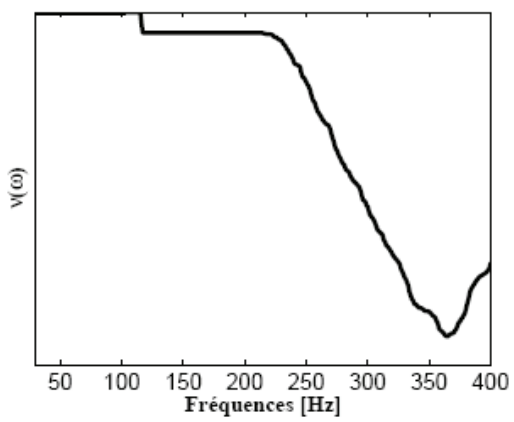

Figure 5: average coefficient of participating mass computed for the dash insulator.

\section{DAMPING COEFFICIENT}

Since damping is mainly due to the soft layer material, its measured loss factor is used for the simplified model.

\section{ACADEMIC VALIDATION}

The proposed theory was first applied to the academic case of a "kind of" RTC3 setup. A flat sample of insulating device $(25 \mathrm{~mm}$ of PU Foam and $4 \mathrm{~mm}$ of EPDM) is laid on a framed flat steel plate. A mechanical excitation is applied and both mean vibrations levels of the supporting plate and trim skin (heavy layer) were measured. Figure 6 shows a picture of the experimental setup. This first application only deals with structural aspects.

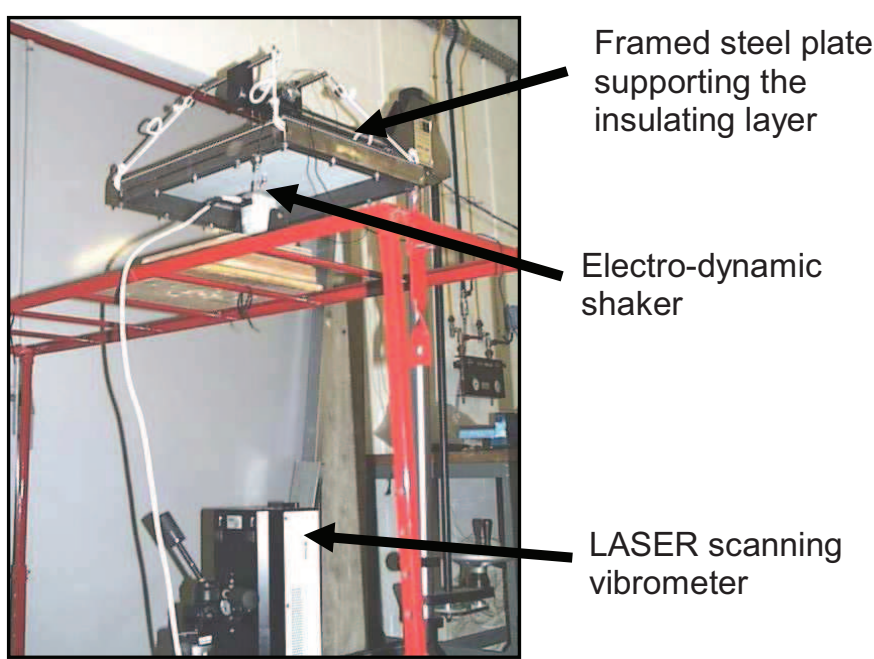

Figure 6: Academic experimental setup

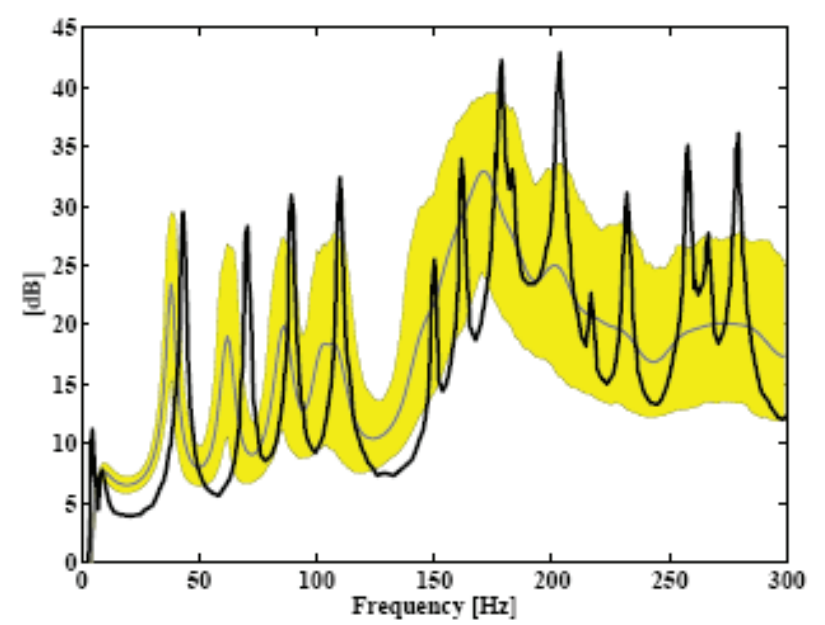

Figure 7: Mean square velocity of the steel plate, without insulating material. Measured value (dark line); mean computed value (grey line); confidence regions of the computations with a $95 \%$ probability (yellow patch).

The experimental setup, without insulating material, have been modeled using a finite element software, using best practice modeling rules, including constant damping, and no updating. A nonparametric probabilistic model is then identified [1] to the measurements, invoking the maximum of likelihood principle. Figure 7 shows the agreement that is obtained between the probabilistic model and the only one existing measurement. This agreement is far from perfect, and correlatively, confidence regions of the stochastic model are large (up to $15 \mathrm{~dB}$ ). This only means our model is poor regarding the sensitivity of the studied structure to small changes.

The fuzzy-structure modeling of the insulating layers was then added to the previous probabilistic model, as explained in the previous sections. Results of the computations are compared to the measurements of the steel plate covered by the trim material on figure 8 . First, one may notice the great changes in the dynamical response, compared to the case of the bare plate. The 
trim has a really strong damping effect on its supporting structure. This effect seems to be perfectly simulated by the proposed model since the agreement is rather good. Surprisingly, the width of the confidence region of the computations is reduced to less than $5 \mathrm{~dB}$. This is interpreted as an effect of damping, which decreases the previously observed sensitivity of the framed steel plate.

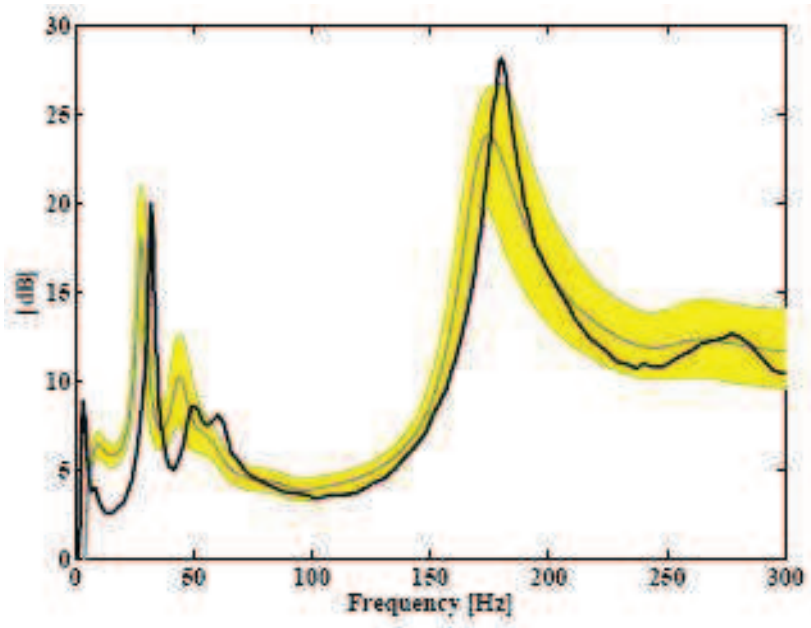

Figure 8: Mean square velocity of the steel plate, covered with insulating material. Measured value (dark line); mean computed value (grey line); confidence regions of the computations with a 95\% probability (yellow patch).

This section's results validate vibratory aspects of the proposed model. Moreover, this first application allowed a good understanding of the physical effects provided by the fuzzy structure theory.

\section{AUTOMOTIVE APPLICATION}

In this last section, the proposed theory is applied to a full vehicle problem [12], with two main objectives. The first one of course is the validation of the proposed modeling.

A second objective is to ensure the industrial application of the proposed model. The main practical difficulty of the proposed method relies in the computation of the spatial coupling matrices $\left(\left[B^{s}\right],\left[B^{a}\right]\right.$ and $\left.\left[C^{s}\right]\right)$. This point was simplified - compared to the standard modeling - by using compatible structural and cavity meshes. Incompatible meshes require specific developments which are in progress. Apart from this point, the FE model and numerical tools are the same as the ones currently used in the industrial process. A nonparametric probabilistic model is later built for the structural-acoustic model with dispersion parameters taken from a previous work $[6,8]$.

Figure 9 shows the model of the car body that was used to perform this application. The model has about 1.2 million degrees of freedom. 1700 structural modes and 140 acoustic modes were extracted up to $600 \mathrm{~Hz}$. Since the trim is modeled by added surface mass in the current model, a modified structural model without these masses was built, prior to the fuzzy structure application. Two different patches of insulating material were considered: one for the dash, the other for the floor. Fuzzy parameters have been computed - according to a previous section - from 3D finite element models shown on figure 10. Only elastic material properties were used.

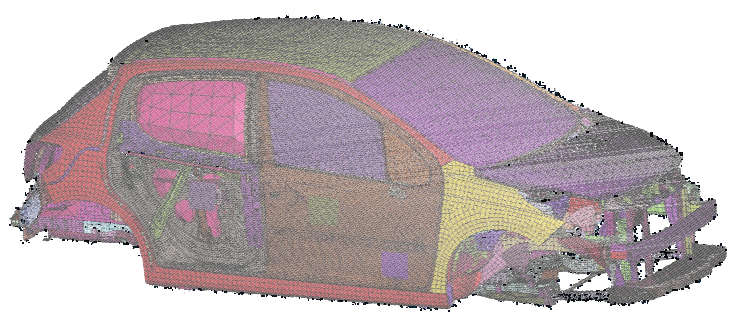

Figure 9: Structural-acoustic model mesh.

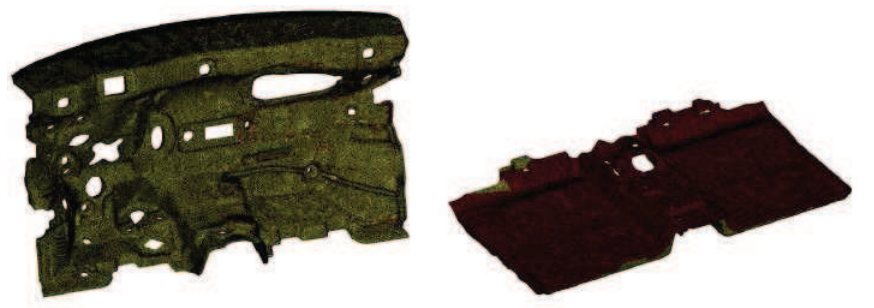

Figure 10: Finite element mesh

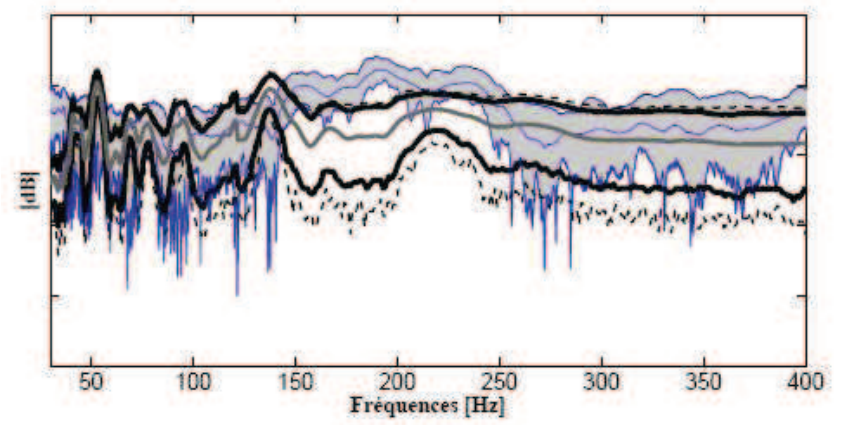

Figure 11: Vibroacoustic FRF between the driver's ear and the right engine mount (TZ). Mean value and 95\% confidence interval. Computation: dark black. Measurement: thin blue.

Since the studied vehicle is in production, dispersion measurements were available, providing a robust basis to evaluate the relevance of the proposed modeling. Vibratory, acoustic and vibroacoustic FRF of 40 vehicles of the same kind have been measured.

Computation results are compared to experiments in the range [30 Hz-400 Hz]; as an example, the mean values and the confidence intervals with a $95 \%$ probability of the vibroacoustic FRF between the driver's ear and the right engine mount are plotted on Figure 11. 
It may be seen that except between $130 \mathrm{~Hz}$ and $250 \mathrm{~Hz}$, the agreement is rather good: the mean value differences are lower than $3 \mathrm{~dB}$ and the spread of experimental and computed results is of the same order of magnitude. Between $150 \mathrm{~Hz}$ and $250 \mathrm{~Hz}$, there are obviously some pre-existing modeling mistakes in the mean model. It is a benefit of the nonparametric modeling to clearly show where large discrepancies lie.

\section{CONCLUSION}

A simplified probabilistic method is proposed to model mass-spring sound insulation devices. It is an application of the fuzzy structure theory, which considers probabilistic distributions of elementary oscillators. The method only addresses the mean value estimations. Fluctuations are later simulated using a nonparametric probabilistic model of the spatial coupling terms associated to the fuzzy structure.

A fuzzy structure patch is defined by his attachment surface, and only three frequency-dependent parameters: participating mass, modal density and damping coefficient. At the moment, these parameters are computed from a separate finite elements model of the considered insulation device.

The method is developed for a modal formulation of structural-acoustic problems, and is implemented as an alteration of the standard numerical procedure. As a consequence, the model can practically be built over any existing vibroacoustic FE model. At the moment, a restriction is that fluid and structural meshes have to be compatible over the fuzzy structure patches extents.

After a first application in an academic case, the proposed model is applied in the case of a real full vehicle vibroacoustic model.

Although the comparison of the computations with experiments seems to be good - apart from initial model discrepancies -, it doesn't fully ensure the model validity; in such a complex case the trim model sensitivity may be strongly dependent on the body modeling - including the modeling errors -, and a complete validation certainly requires deeper investigations.

\section{REFERENCES}

1. Fernandez, C., Soize, C. and Gagliardini, L. (2009). "Fuzzy structure theory modeling of sound-insulation layers in complex vibroacoustic uncertain sytems theory and experimental validation", Journal of the Acoustical Society of America Accepted for publication in 2008.

2. Soize, C. (1986), "Probabilistic structural modeling in linear dynamical analysis of complex mechanical systems. I. Theoretical elements", Rech. Aerosp. 5, 23-48. (English edition).

3. Ohayon, R. and Soize, C. (1998). Structural Acoustics and Vibration, Mechanical Models
Variational Formulations and Discretization (Academic press, San Diego, USA).

4. Pierce, A. D., Sparrow, V. W. and Russell, D. A. (1995). "Fundamental Structural-Acoustic Idealizations for Structures with Fuzzy Internals", Journal of Vibration and Acoustics 117, 339-348.

5. Soize, C. (2003). "Random matrix theory and nonparametric model of random uncertainties in vibration analysis", Journal of Sound and Vibration 263, 893-916.

6. Durand, J.-F., Gagliardini, L. and Soize, C. (2008). "Structural-acoustic modeling of automotive vehicles in presence of uncertainties and experimental identification and validation", Journal of the Acoustical Society of America 24, 1513-1525.

7. Durand, J.-F., Gagliardini, L. and Soize, C. (2004). "Nonparametric modeling of the variability of vehicle vibroacoustic behavior", in Proceedings of the SAE 05, Noise and Vibration Conference and Exhibition, 16-19 May, CDROM ISBN 0-7680-1657-6 (Traverse City, Michigan, USA).

8. Durand, J.-F. (2007). Modélisation de Véhicules en Vibroacoustique Numérique avec Incertitudes de Modélisation et Validation Expérimentale. Thèse de doctorat, Université de Marne-la-Vallée.

9. Hamdi, M. A., Zhang, C., Mebarek, L., Anciant, M. and Mathieux, B. (2005). "Analysis of vibroacoustic performances of a fully trimmed vehicle using an innovative sub-system solving approach faciliting the cooperation between carmakers and sound-package suppliers", in Proceedings of EURODYN05, International Conference in Structural Dynamics, 4-7 September, CDROM ISBN 90-5966-033-1 (Paris, France).

10. Coyette, J., Lielens, G., Van den Nieuwenhof, B., Bertolini, C., Gaudino, C., Misaji, K. and Ide, F. (2007). "From body in white to trimmed body models in the low frequency range : a new modeling approach", in Proceedings of SAE 07, Noise and Vibration Conference and Exhibition, 15-17 May, CDROM ISBN (St. Charles, Illinois, USA).

11. Van den Nieuwenhof, B., Lielens, G., Coyette, J., Acher, F. and d'Udekem, D. (2008). "Efficient analysis of large trimmed configurations using modal approaches", in Proceedings of ISMA08, International Conference on Modal Analysis Noise and Vibration Engineering, 15-17 September, CDROM ISBN 9789073802865 (Leuven, Belgium).

12. Fernandez, C. (2008). Modélisation et validation expérimentale des complexes insonorisants pour la prévision vibroacoustique numérique basse et moyenne fréquences des automobiles. Thèse de doctorat, Université Paris-Est, France.

\section{CONTACT}

laurent.gagliardini@mpsa.com 
